\title{
ANOMALÍAS DE GAS RADÓN ASOCIADAS A LA SISMICIDAD EN LA REGIÓN VOLCÁNICA DE LOS COCONUCOS (CAUCA, COLOMBIA)
}

\author{
RADON GAS ANOMALIES ASSOCIATED WITH THE SEISMICITY \\ IN THE VOLCANIC REGION OF THE COCONUCOS (CAUCA, COLOMBIA)
}

\author{
Luisa F. Meza', John M. Londoño², Rosa L. Alpala³, Andrés Narváez ${ }^{4}$ \\ *Email de correspondencia: Imeza@sgc.gov.co
}

Recibido: 28/08/2017

Aceptado: 03/11/2017

Publicado en línea: 20/04/2018

Citación: Meza, L. F., Londoño, J., Alpala, R. L. y Narváez, A. (2018). Anomalías de gas radón asociadas a la sismicidad en la región volcánica de los Coconucos (Cauca, Colombia). Boletín Geológico, 44, 101-114.

\section{RES U M E N}

Se llevó a cabo un estudio de la relación entre la sismicidad de fractura y las emisiones de radón $\left({ }^{222} \mathrm{Rn}\right)$ en suelo en la región volcánica de los Coconucos, usando tres estaciones isotópicas ubicadas en el área de influencia de fallas activas en el período 2006-2013.

Para este estudio se definieron anomalías de gas radón como aquellos valores de concentración por encima del promedio más la mitad de la desviación estándar. Estos valores se correlacionaron con sismos de magnitudes locales (ML) mayores a 2,8, y se llegó a la conclusión de que valores por encima de $2300 \mathrm{pCi} / \mathrm{L}$ estuvieron asociados a la ocurrencia posterior de estos sismos en un radio de $35 \mathrm{~km}$ del centro de la red de estaciones. El rango de tiempo entre la detección de la anomalía y la ocurrencia del sismo (ML > 2,8) varió entre 3 y 84 días, con un promedio de 31 días. De un total de 15 sismos ocurridos en el período de estudio con $\mathrm{ML}>2,8$, trece fueron asociados con anomalías de gas radón, para una probabilidad de detección del 87 \%. Esto permite establecer que la medición de concentración de gas radón en el suelo, usando una distribución apropiada de estaciones, es una herramienta útil para el pronóstico de sismicidad posterior en la zona de los Coconucos.

Palabras clave: sismicidad, gases difusos (222Rn), cadena volcánica los Coconucos.

\footnotetext{
M. Sc. en Química. Observatorio Vulcanológico y Sismológico de Popayán del Servicio Geológico Colombiano

Ph. D. en Geofísica. Observatorio Vulcanológico y Sismológico de Popayán del Servicio Geológico Colombiano

3 Especialista en Sistemas de Información Geográfica. Observatorio Vulcanológico y Sismológico de Popayán del Servicio Geológico Colombiano

4 Geógrafo. Observatorio Vulcanológico y Sismológico de Popayán del Servicio Geológico Colombiano
} 


\begin{abstract}
A B S T R A C T
A study of the correlation of seismicity with radon in soil emissions $\left({ }^{222} \mathrm{Rn}\right)$ was carried out at Coconucos volcanic region by using three isotopic stations located close to geological faults for the period of 2006-2013.

In this study, a radon anomaly was considered as those values above the average plus half of the standard deviation. These radon anomalies were correlated with earthquakes with a local magnitude (ML) greater than 2.8 , concluding that radon anomalies above $2300 \mathrm{pCi} / \mathrm{L}$ were associated with a further occurrence of earthquakes with $\mathrm{ML}>2.8$ for a radius of $35 \mathrm{~km}$ taken from the center of the radon stations network. The difference in time between the detection of the radon anomaly and the occurrence of the earthquake ranged from 3 to 84 days, with 31 days average. From a total of 15 local earthquakes with ML $>2.8$ that occurred during the studied period, 13 were associated with a previous radon anomaly, resulting in a probability of detection of $87 \%$. These results suggest that the regular measuring of radon in soil using a well-established network of isotopic stations is a good tool for forecasting the seismicity in the Coconucos region.
\end{abstract}

Keywords: seismicity, diffuse gas (222Rn), Coconucos volcanic chain.

\section{INTRODUCCIÓN}

1 gas radón, con su isótopo ${ }^{222} \mathrm{Rn}$, por ser un elemento cuya producción es continua en el interior de la corteza terrestre, tiende a escapar de las rocas donde se genera y a incorporarse a los sistemas fluidos, en los que se ha observado que su concentración puede variar en relación con el desarrollo de actividad tectónica y volcánica (Gasparini y Mantovani, 1978; King, 1980, 1985; Wakita et al., 1988, Martinelli y Ferrari, 1991; Connor et al., 1996; Heiligmann et al., 1997; Nishizawa et al., 1998).

El gas radón no reacciona con otros elementos y compuestos, y, de acuerdo con su mecanismo de difusión, se libera frecuentemente por las fallas $\mathrm{u}$ otras estructuras del subsuelo. Por sus características físicas, su detección se facilita en fisuras, fallas geológicas y flujos de agua subterránea. El isótopo ${ }^{222} \mathrm{Rn}$ tiene un tiempo de vida media favorable de 3,82 días, y es el isótopo más abundante, lo que lo convierte en el más útil para estudios geohidrológicos, debido a que las estructuras geológicas y la actividad de las mismas alteran la permeabilidad del suelo, por lo que se puede aprovechar el comportamiento del gas para registrarlo en la superficie. Por esto, se puede inferir que la concentración del ${ }^{222} \mathrm{Rn}$ se encuentra asociada a zonas donde se presenta un tectonismo activo, razón por la que los sectores en los que se realizan las mediciones continuas de las emisiones del isótopo radiactivo ${ }^{222} \mathrm{Rn}$ están localizados principalmente sobre fallas activas dentro del complejo volcánico, así como sobre zonas en las que existe una alta probabilidad de movimiento de fluidos y generación de fracturas.

Hay otros procesos dinámicos que pueden producir un incremento del flujo de radón en la superficie: la microfracturación de rocas inducida por esfuerzos causados por eventos intrusivos (Thomas et al., 1986), así como el aumento del flujo térmico o de las descargas de vapor (Cox, 1980; Cox et al., 1980; De la Cruz Reina et al., 1985).

Además, se debe considerar que el radón no tiene una movilidad propia y necesita de un gas transportador que le permita moverse con rapidez y a grandes distancias. En los sistemas naturales, el dióxido de carbono $\left(\mathrm{CO}_{2}\right)$ y el agua son los principales transportadores de este gas.

Las emisiones de gas radón han sido usadas desde hace décadas para la predicción de sismos (Ulomov y Mavashev, 1971; King, 1980; Teng, 1980; Shapiro et al., 1980; Fleisher y Mogro, 1985; Thomas et al., 1986; Wakita et al., 1988; Virk y Singh, 1993). Asimismo, se han utilizado en la prospección de uranio (Fleischer et al., 1980; Ramola et al., 1988), hidrocarburos (Garzón, 1987; Mazadiego, 1994) y campos geotérmicos (Cox, 1980; Whitehead et al., 1983; Segovia et al., 1991). También han sido empleadas en la detección de sistemas de fallas o fracturas (Crenshaw et al., 1982; Aubert y Baubron, 1988; Fuentes et al., 1995), en zonas afectadas por deslizamientos (Purtscheller et al., 1994; Purtscheller et al., 1995), como trazador atmosférico (Junge, 1963; Guedalia et al., 1980), en la determinación 
del sistema de circulación y ventilación en minas y cuevas (Wilkening y Watkins, 1976; Hunyadi et al., 1991), y en hidrogeología (Berelson et al., 1982; Cable et al., 1996). Por otra parte, se han realizado estudios sobre la relación de emisiones de gas radón y actividad volcánica en algunos volcanes activos, como en Vulcano (Italia) (Del Pezzo et al., 1981; Cioni et al., 1984; Baubron et al., 1990, 1991; Toutain et al., 1992), el Kiillauea (Hawái, EE. UU.) (Cox et al., 1980; Cox y Cuff, 1981; Thomas et al., 1986), el Krafla (Islandia) (Hauksson, 1981), el Etna (Italia) (Seidel y Monnin, 1984; Baubron et al., 1991; Parello et al., 1995; Chiodini et al., 1996; D’Alessandro y Parello, 1997), el Pitón de la Fournaise (isla Reunión, Francia) (Seidel et al., 1988), el Izu-Oshima (Japón) (Sato et al., 1992), el cerro Negro (Nicaragua) (Connor et al., 1996), el Galeras (Colombia) (Heiligmann et al., 1997), el volcán Nevado del Ruiz y cerro Machín (Colombia) (Londoño, 2009), entre otros.

Este estudio está enfocado en analizar las variaciones temporales de emisión de gas radón en el suelo y su relación con la actividad sísmica en el área volcánica de los Coconucos (Cauca, Colombia) por ser una zona donde confluyen tanto fallas como volcanes activos.

\section{MetodologíA}

Para las mediciones de gas radón se utilizó el sistema de cámaras ionizantes E-PERM (Electret-Passive Environmental Radon Monitor) de Kotrappa et al. (1988), consistente en electretos de larga duración con cargas superiores a $700 \mathrm{~V}$, que se insertan en una cámara de $35 \mathrm{~mL}$. Este sistema se pone dentro de un tubo de PVC abierto en la parte inferior, que se entierra en el suelo a una profundidad de aproximadamente $1,7 \mathrm{~m}$, y se cubre con una tapa en la parte superior para evitar el escape de gas radón; de esta manera sirve como una trampa de captura de gas radón. El tiempo de medición de cada dato fue de diez días, aproximadamente. Una vez cumplidos los diez días de cada medición, el sistema E-PERM se retira para hacer las mediciones de la descarga en voltaje de los electretos usando un equipo SPERM-1 (Kotrappa et al., 1990).

Los electretos se cambian y se inicia un nuevo ciclo de medición en cada una de las estaciones. Usando las correcciones apropiadas de dosis de radiación gamma, altitud y factores de calibración de fábrica, la concentración de gas radón en cada estación, según Garzón (1998) modificado de Kotrappa et al. (1988-1990), se obtiene mediante la siguiente ecuación:

$[R n]=\frac{V_{i}-V_{f}}{F C^{\star} T}-D G$

Donde

$[R n]=$ concentración de gas radón en $\mathrm{pCi} / \mathrm{L}$

$V_{i}, V_{f}=$ voltaje inicial y final del electreto en voltios

$T=$ tiempo de exposición en días

$F C=$ constante $\mathrm{o}$ factor de calibración en $\mathrm{pCi} / \mathrm{L}$

$D G=$ dosis de radiación gamma ambiental en el sitio donde se realiza la medición. Para el caso de este estudio se tomó el valor de 1 .

\section{ERrores en LAS MEdiciones de GAS RadóN}

Las inexactitudes de cálculo en las mediciones del radón mediante el sistema E-PERM pueden dividirse en errores debidos a las imperfecciones geométricas de los electretos y de las cámaras. Existe una incertidumbre con respecto al volumen de las cámaras y al grosor de los electretos relacionada con posibles inconsistencias en su producción en serie. De acuerdo con el inventor, se determinó experimentalmente que este error (E1) es del $5 \%$, así:

$\mathrm{E} 1= \pm 0,05(\mathrm{Vi}-\mathrm{Vf}) /\left(\mathrm{FC}{ }^{\star} \mathrm{D}\right)$

Otra fuente de error son aquellas fallas provenientes de la lectura del potencial inicial y final del electreto. La diferencia máxima entre dos medidas puede alcanzar el valor de 1,4 voltios. El error fraccional (EF), asociado con la diferencia de dos lecturas, es

$\mathrm{EF}= \pm(2) 1 / 2 /(\mathrm{Vi}-\mathrm{Vf})$

El error de la concentración E2 es

$\mathrm{E} 2= \pm(2) 1 / 2 / \mathrm{FC} * \mathrm{D}$

Teniendo en cuenta los errores E1 y E2, los correspondientes a las mediciones de las alturas donde se ubican las estaciones, y los errores en las mediciones de las dosis de radiación gamma ambiental, el error total asociado a las mediciones de radón estuvo por debajo del $12 \%$. 


\section{Anomalías de gas radón}

Para la determinación de anomalías de gas radón se siguió la metodología de Londoño (2013), que define una anomalía de radón como

$A_{R n}=[R n]_{\max }+\frac{\sigma}{2}$,

Donde

$\mathrm{A}_{\mathrm{Rn}}=$ anomalía de gas radón

$[R n]_{\text {máx }}=$ concentración máxima de las anomalías de cada medición en la red de estaciones

$\sigma=$ desviación estándar

Por lo tanto, esta metodología considera como anomalía los valores de concentración de gas radón por encima del promedio más la mitad de la desviación estándar en un grupo de estaciones seleccionadas apropiadamente.

\section{Datos y procesamiento}

De una red de 33 estaciones isotópicas dedicadas al monitoreo de radón en la zona volcánica de los Coconucos, se seleccionaron aquellas que tuvieran mayor número de datos, mayores variaciones en los valores de radón, mayor período de muestreo y, en lo posible (aunque no necesariamente), mayor cercanía a zonas falladas de acuerdo con la metodología de Londoño (2013). Después de varias pruebas de ensayo y error teniendo en cuenta grupos de estaciones que cumplieran con los criterios mencionados, se seleccionaron tres estaciones: Agua Tibia (AT); Agua Hirviendo (AH) Y Tabio (TB). Con las series de tiempo de las estaciones escogidas, correspondientes al período 2006-2013, se calcularon promedios mensuales de concentración de gas radón y valores máximos por cada medida en la red de estaciones seleccionadas (Londoño, 2013). La figura 1 muestra la localización del área de estudio, la red de estaciones isotópicas y las estaciones seleccionadas para el estudio.

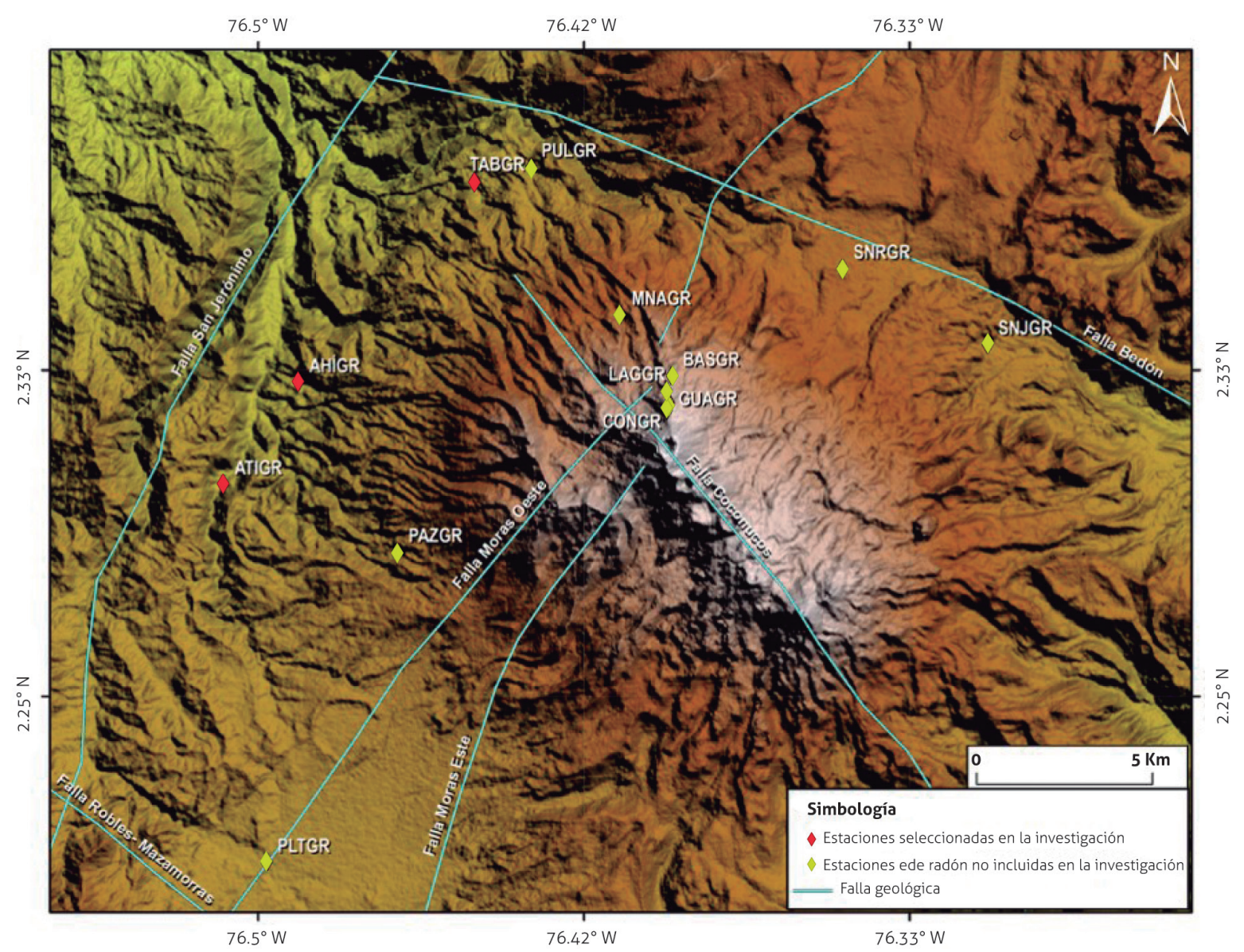

Figura 1. Localización del área de estudio. Red de estaciones isotópicas de ${ }^{222} \mathrm{Rn}$ Fuente: autores 
Para la comparación de la sismicidad, se utilizó la base de datos sísmica del Servicio Geológico Colombiano (SGC) y del Observatorio Vulcanológico y Sismológico de Popayán (OVSPO) correspondiente al período 2006-2013. Se seleccionaron inicialmente los sismos tanto volcano-tectónicos (VT) como tectónicos locales con magnitudes locales (ML) mayores a 1,5, localizados en un radio de $35 \mathrm{~km}$ del centro de la red de radón, de acuerdo con el criterio de Dobrovolsky et al. (1975) y Fleischer (1988). Las figuras 2a y 2b muestran la localización epicentral de los sismos seleccionados y las curvas de energía sísmica liberada, así como el número de sismos por día para los sismos con una ML mayor a 1,5 en el período 2006-2013 en la zona de los Coconucos.

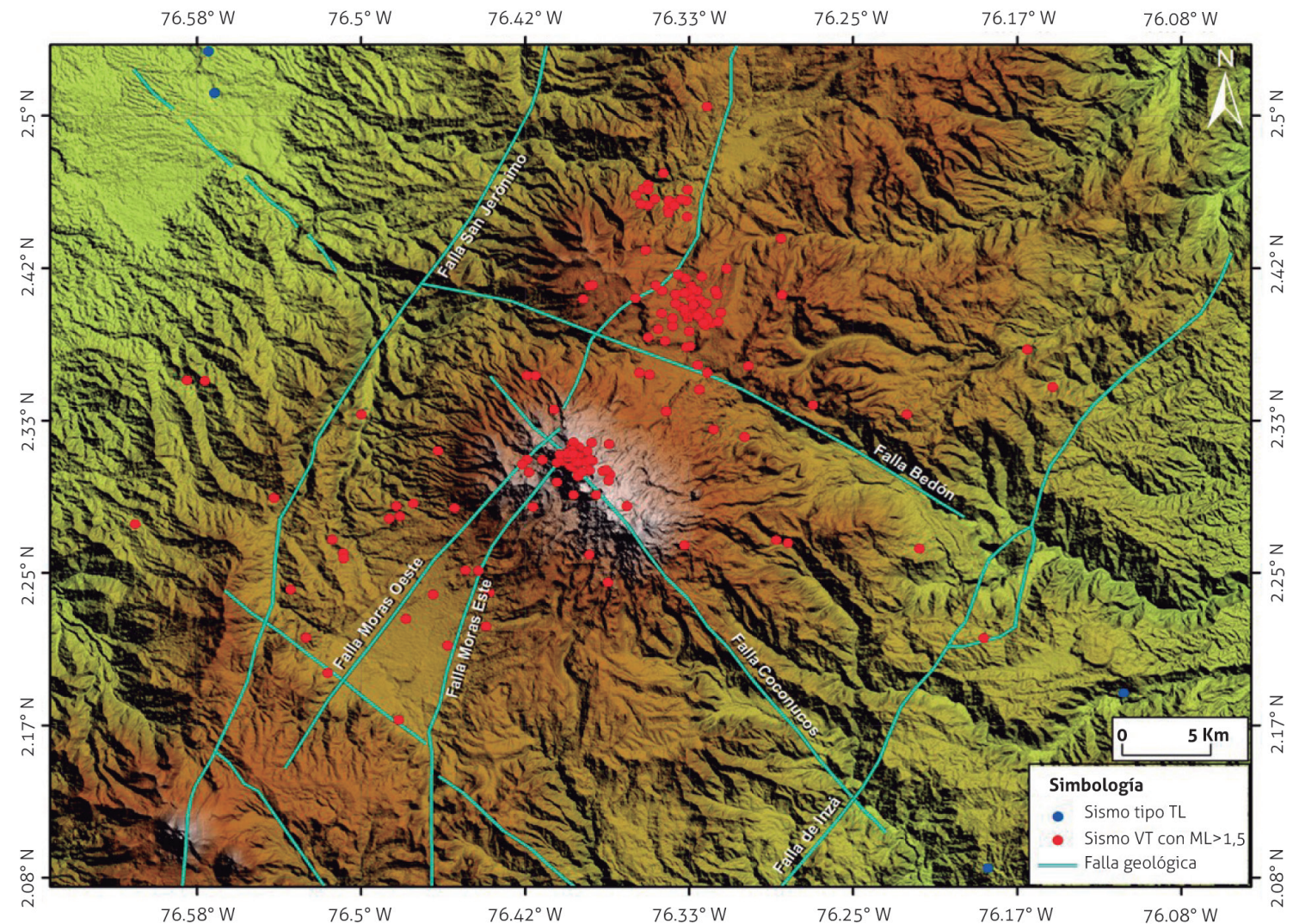

Figura 2a. Localización hipocentral de la sismicidad seleccionada, ML > 1,5 (2006-2013)

Fuente: autores

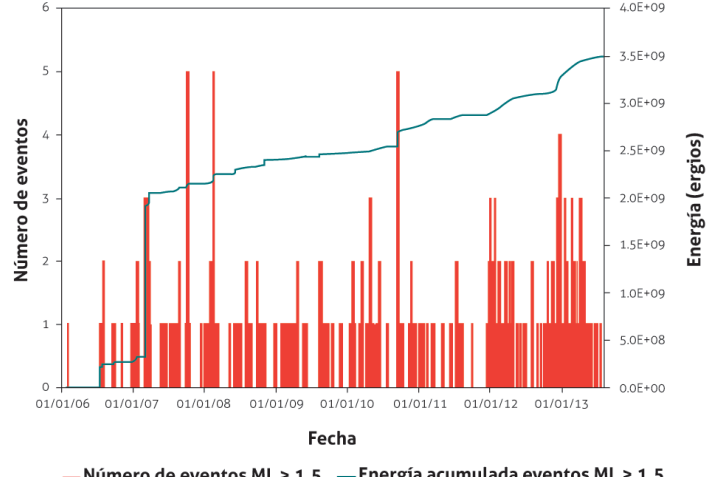

-Número de eventos $M L>1,5$-Energía acumulada eventos $M L>1,5$ Figura 2 b. Número de eventos y energía acumulada en eventos con ML > 1,5 (2006-2013)

Fuente: autores

\section{Contexto geológico estructural de la región VOLCÁNICA DE LOS COCONUCOS}

La cadena volcánica de los Coconucos está compuesta por quince centros eruptivos alineados con una orientación $\mathrm{N} 39^{\circ} \mathrm{W}$, la mayoría de ellos sobre el trazo de la falla Coconucos. Entre estos centros eruptivos se destaca el volcán Puracé, que es un estratovolcán activo (4650 msnm), localizado a una distancia de $26 \mathrm{~km}$ al SE de la ciudad de Popayán. Es el extremo noroccidental de la cadena volcánica de los Coconucos. La actividad fumarólica se concentra en el interior del cráter, principalmente 
en una gran grieta que atraviesa el fondo; también hay un notable campo fumarólico en el flanco externo NW del

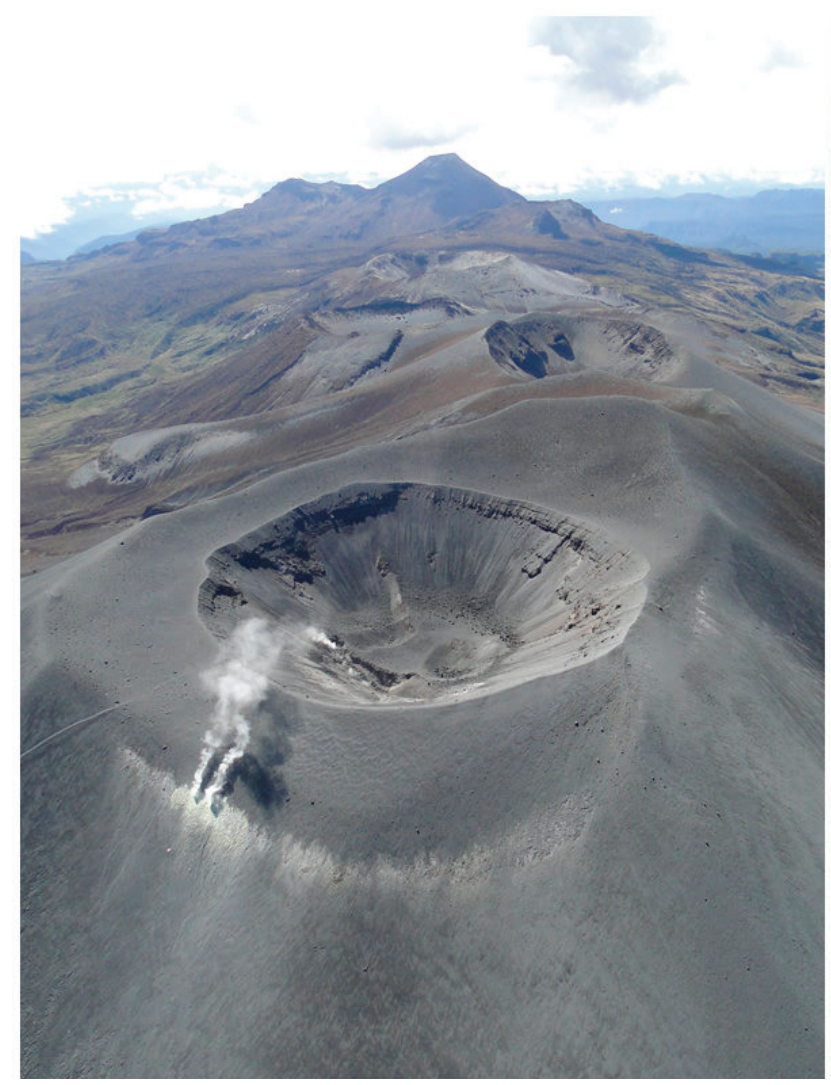

volcán (fumarola lateral) (Monsalve y Pulgarín, 1993). Véase la figura 3.

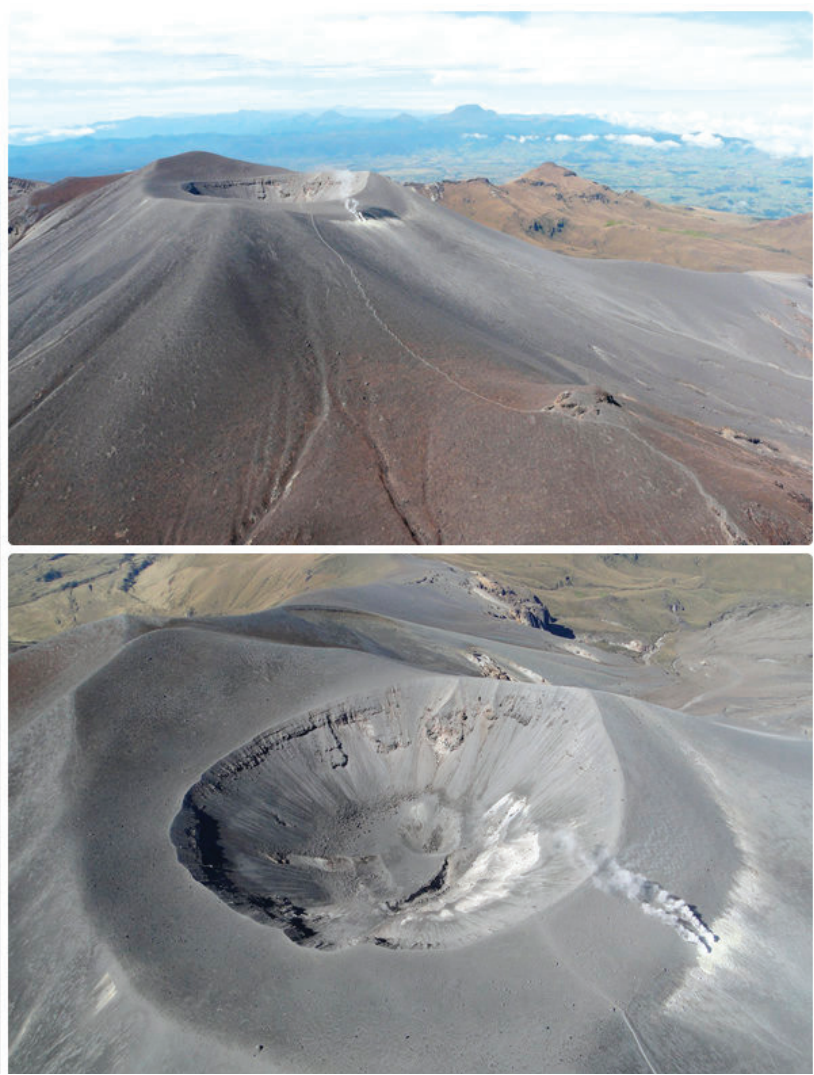

Figura 3. Cadena volcánica de los Coconucos. En primer plano se observa al volcán Puracé. Imágenes tomadas durante el sobrevuelo realizado el 11 de octubre del 2011

Fuente: SGC-OVSPOP

Estudios geológicos recientes revelan una historia eruptiva caracterizada por actividad predominantemente explosiva, cuyos productos principales han sido lavas andesíticas, flujos piroclásticos, piroclastos de caída y flujos de lodo (Monsalve y Pulgarín, 1993). Esta actividad ha sido reportada a partir de 1801, y se han descrito, desde 1827 , al menos quince episodios eruptivos bien documentados que han generado daños materiales y pérdida de vidas. La última erupción menor considerada ocurrió en marzo de 1977, asociada con salida de cenizas. No se sabe de actividad reciente de otros volcanes. La cadena volcánica de los Coconucos (CVLC) se encuentra afectada por tres sistemas de fallas regionales con direcciones principales NESW, NW-SE y NNE, (Monsalve y Pulgarín, 1999), de las cuales se identifican las siguientes (figuras 2 a y $2 b$ ):
Falla Moras. De acuerdo con Orrego (1977), esta falla tiene una estructura de rumbo dextral, con ángulo alto y está inclinada al este. Asimismo, Orrego y París (1991) consideran que este sistema representa una sutura paleozoica que sirve de límite entre las rocas metamórficas paleozoicas del complejo Cajamarca y del neis de Quintero. Según los mismos autores, esta sutura se encuentra alineada con la cadena volcánica actual de la cordillera Central, y posiblemente corresponde con la megafalla $\mathrm{Pa}$ lestina del centro del país.

Falla Bedón. Tiene una tendencia noroccidental. En el área de estudio se evidencia la expresión de esta falla con un rasgo lineal en un segmento entre los ríos San Francisco y Bedón (figuras 2a y 2b). 
Falla Coconucos. La falla Coconucos es una estructura con dirección NW-SE, a lo largo de la cual se alinean los volcanes de la CVLC, desde el Puracé, en el noroccidente, hasta el Pan de Azúcar, en el suroriente. Según Gil (1998), en la intersección de esta falla con la falla de Moras se ha detectado una fuente sismogénica denominada fuente sismogénica Puracé. Pulgarín et al. (1994 en prensa).

Falla Inzá. La falla Inzá fue cartografiada por Instituto Colombiano de Energía Eléctrica (ICEL) en 1983, y su nombre proviene de la población de Inzá (Cauca). También es conocida como falla Moras y se la consideran parte de un sistema de fallas de rumbo que corta las fallas con dirección NE-SW de la parte alta de la cuenca del río Páez; se la considera de tipo inverso y con inclinación del plano al oeste. Por afectar al complejo Cajamarca, se la considera una falla antigua que ha contribuido al levantamiento de la cordillera Central en el flanco este.

Presenta un rumbo $\mathrm{N} 30^{\circ}$ a N $45^{\circ} \mathrm{E}$, y sirve como límite entre las rocas paleozoicas del complejo Cajamarca con rocas cretácicas y jurásicas pertenecientes a las sedimentitas Santa Leticia, las arenitas blancas del cerro Tabor y al batolito de Ibagué.

Falla San Jerónimo. Es la falla más oriental del sistema de fallas de Romeral definido por Grosse (1926), y propuesto por Orrego y París (1991) para denominar las fallas que se localizan en el flanco occidental de la cordillera Central, con dirección preferencial NNE. Marquínez et al. (2003), citando a otros autores, la describe como una estructura regional que limita el complejo Cajamarca, de edad paleozoica y afinidad continental, con el complejo Quebrada Grande, de edad mesozoica y afinidad oceánica. Esta estructura se considera inversa, y tiene una dirección aproximada N $37^{\circ}$ E, que buza hacia el E. Del análisis de fotografías aéreas e imágenes satelitales de la zona afectada por la falla San Jerónimo puede interpretarse que esta marca el borde occidental de la caldera de Paletará, lo que constituye un argumento de peso para la definición de dicha caldera como caldera de colapso.

A esta falla se han asociado varios de los sismos sentidos en un área amplia del sur del departamento del Cauca, como los ocurridos los días 7 de enero y 2 de febrero de 2003 (Pulgarín y Agudelo, 2003), además de otros sismos sentidos en la región de Paletará y Paispamba en años recientes, de acuerdo con los datos de la red de monitoreo volcánico del Observatorio Vulcanológico y Sismológico de Popayán.

\section{Resultados}

Antes de analizar los resultados de las mediciones de gas radón y relacionarlos con la sismicidad, es necesario determinar si dichos resultados están afectados por algunas variables que pueden modificar en parte la tendencia de los datos. Uno de los factores que más afectan los valores de gas radón es el clima. La figura 4 muestra las variables atmosféricas, de precipitación, presión atmosférica y temperatura ambiente, y su relación con las emisiones de gas radón en las estaciones seleccionadas (máxima concentración por medida). De acuerdo con los resultados obtenidos, se concluyó que no hay una correlación clara entre las variaciones temporales de gas radón y cambios atmosféricos en el período estudiado. Por tal razón, es posible atribuir las variaciones temporales de gas radón a fenómenos diferentes a los atmosféricos.

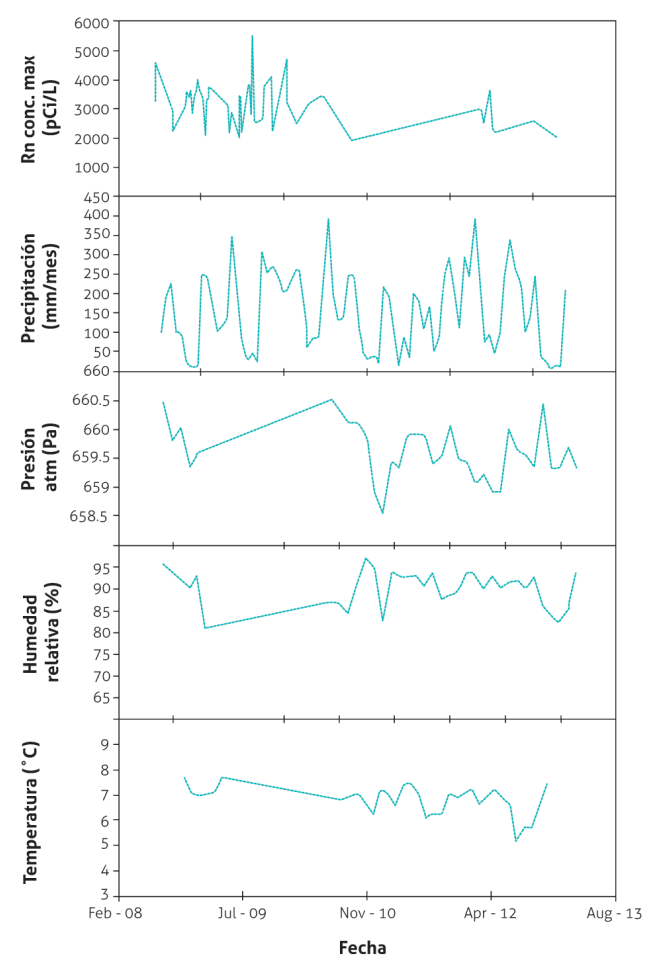

Figura 4. Relación de emisiones máximas de gas radón por medida $v s$. Promedios mensuales de precipitación, presión atmosférica, humedad y temperatura ambiente. Los datos meteorológicos corresponden a la estación Puracé del Ideam

Fuente: autores 
La figura 5 muestra las series de tiempo de las estaciones escogidas para el análisis. De esta figura se puede concluir que en algunos períodos de tiempo hay una correlación clara entre las diferentes estaciones, mientras que, en otras épocas, no la hay.

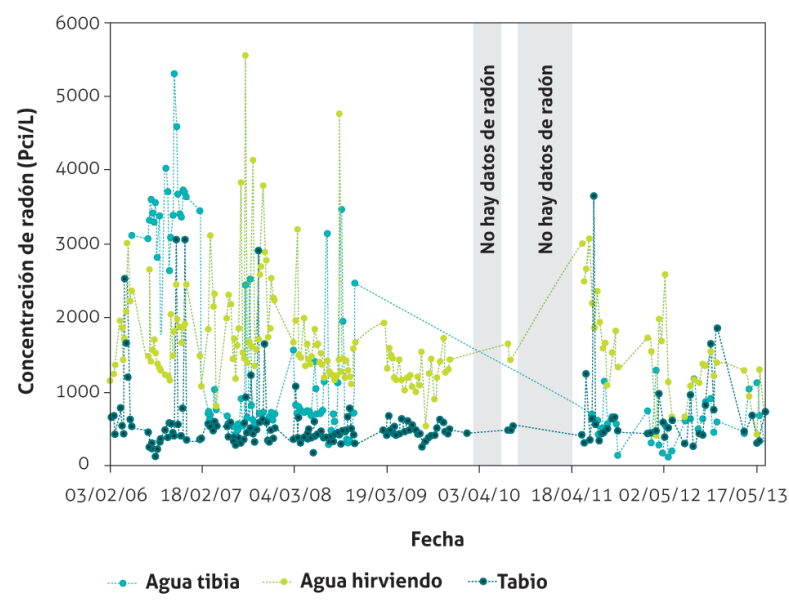

Figura 5. Series de tiempo de las estaciones isotópicas seleccionadas, 2006-2013

Fuente: autores

La figura 6 muestra la concentración máxima de gas radón por cada medida en todas las estaciones y las magnitudes de los sismos con ML > 1,5. A simple vista no se observa una correlación clara entre cambios temporales de concentración de gas radón y ocurrencia de sismos con $\mathrm{ML}>1,5$ en la zona de estudio.

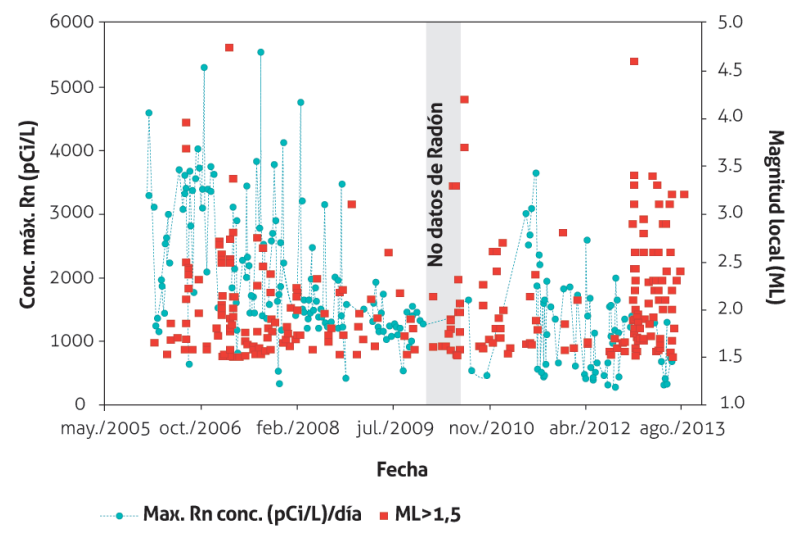

Figura 6. Concentración máxima de gas radón por cada medida, y las magnitudes de los sismos con ML > 1,5

Fuente: autores
Para poder detectar con mayor precisión anomalías de gas radón, y asociarlas con ocurrencia de sismos en la zona de estudio, se procedió a seleccionar solo los valores anómalos, aplicando la ecuación 5. Posteriormente, se realizó un proceso iterativo de pruebas de ensayo y error para determinar el umbral de magnitud a partir del cual los sismos estuvieran asociados con esas anomalías de gas radón, dado que muchos sismos con magnitudes menores a la magnitud umbral no lo están. Por esta razón, se requiere determinar la magnitud menor de detección o asociada con la anomalía (Londoño, 2013). Para este estudio, las anomalías de gas radón correspondieron a valores por encima de $2300 \mathrm{pCI} / \mathrm{L}$. Según los resultados del proceso iterativo de prueba y error, el umbral de magnitud local (ML) que puede ser detectado con esta anomalía es de 2,8 .

De un total de quince sismos con $M L>2,8$ ocurridos durante el período estudiado en un radio de $35 \mathrm{~km}$ con centro en el cráter, trece fueron detectados en un lapso de tiempo de 3 a 84 días después de la anomalía, para una probabilidad de detección del $87 \%$. Un sismo ocurrió en una época en que no se tuvieron datos de gas radón (mayo de 2010), por lo que no se sabe si estuvo asociado o no a una anomalía. Si este sismo se descarta, y se consideran catorce sismos ocurridos durante el período de medición con $\mathrm{ML}>2,8$, en los que hubo datos de gas radón, la probabilidad de detección aumenta al $93 \%$.

La figura 7 muestra la serie de tiempo de las anomalías de gas radón y los sismos con $\mathrm{ML}>2,8$. En esta figura se puede observar que algunos sismos no fueron detectados, si se considera la anomalía mayor a $2300 \mathrm{pCi} / \mathrm{L}$. De acuerdo con la metodología de Londoño (2013), es posible detectar otros cambios cuando la variación de una medida de radón respecto a la siguiente aumenta por encima de un valor determinado, que varía de una región a otra. De acuerdo con este criterio, si se analiza en detalle la serie de datos, se evidencian cambios significativos en los valores máximos de gas radón entre una medida y la siguiente, del orden de $1000 \mathrm{pCi} / \mathrm{L}$, lo que hace suponer que la combinación, tanto de anomalías como de cambios mayores de $1000 \mathrm{pCi} / \mathrm{L}$ entre una medida y otra, definen mejor el cambio en concentración de gas radón asociado a la posterior ocurrencia de un sismo con $\mathrm{ML}>2,8$ en la zona de los Coconucos. 


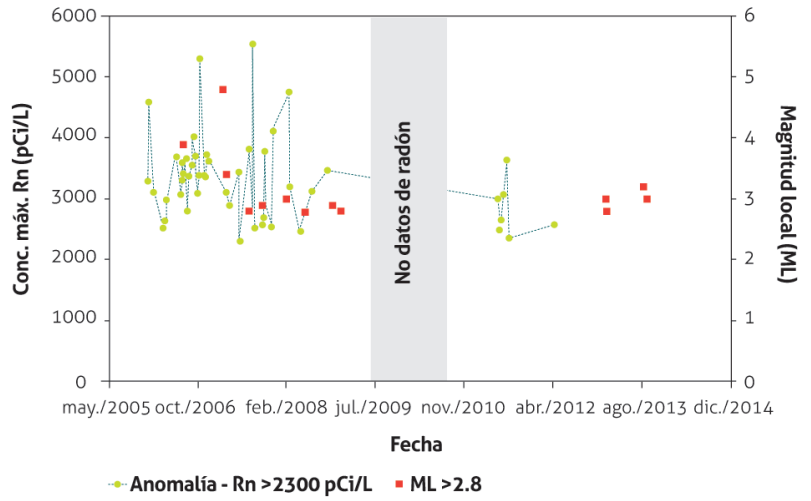

Figura 7. Relación de las anomalías de gas radón $(>2300$ pCi/L) con sismos ML $>2,8$ en la zona de los Coconucos

Fuente: autores

La figura 8 muestra tanto los valores anómalos como los valores máximos de gas radón asociados a cambios significativos entre una medida y otra, y los sismos con magnitudes $\mathrm{ML}>2,8$. También se observa que todos los sismos son detectados apropiadamente. Adicionalmente, en la figura 9 se analiza la relación entre la distancia focal y la anomalía de gas radón, y se encuentra que no existe una correlación clara entre estas dos variables. Esto implica que las anomalías de gas radón detectadas son independientes tanto de la magnitud de los sismos como de la cercanía a la red de detección.

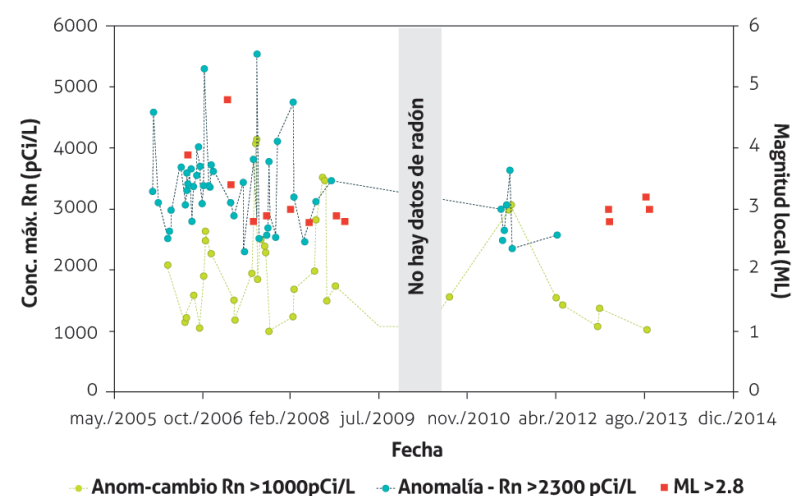

Figura 8. Relación de las anomalías de gas radón $(>2300 \mathrm{pCi} / \mathrm{L})$ y variaciones entre medidas superiores a $1000 \mathrm{pCi} / \mathrm{L}$ con sismos $\mathrm{ML}>2,8$ en la zona de los Coconucos

Fuente: autores

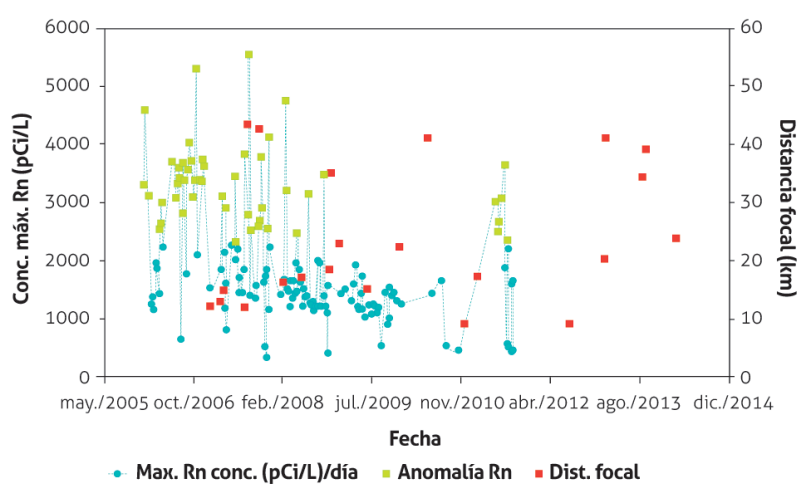

Figura 9. Relación entre la distancia focal de sismos con $\mathrm{ML}>2,8$ y anomalías de gas $\mathrm{Rn}$, y máxima concentración por medida en la zona de los Coconucos

Fuente: autores

\section{Distribución espacial de anomalías de gas radón}

Con miras a determinar espacialmente las zonas con mayor liberación de gas radón, se elaboró un mapa de contornos con los valores máximos de la red de 33 estaciones en la zona de los Coconucos (figura 10). En esta gráfica se tomaron los máximos valores, y queda en evidencia que el valor promedio o base está por debajo de los 731 $\mathrm{pCi} / \mathrm{L}$, lo que quiere decir que las zonas de menor desgasificación están sobre la influencia de la falla Bedón, y que esta se encuentra localizada en una zona distal hacia el sector occidental. Al comparar la desgasificación con las fallas o lineamientos presentes en el área, se deduce que la falla San Jerónimo (SW-W) es la posible fuente principal de emisión de gas radón en el área, además de ser una de las mayores fuentes de emisión de flujo de $\mathrm{CO}_{2}$ en suelo (Meza et al., 2017). Es importante destacar que en cercanías de esta zona anómala se encuentra un campo hidrotermal activo con altos caudales y altas temperaturas, que puede ser también responsable de las mayores liberaciones de gas radón. Por otra parte, los sismos tectónicos locales más distales muy probablemente estén asociados con otras fallas regionales que no fueron cubiertas por la red de monitoreo usada en este estudio. 


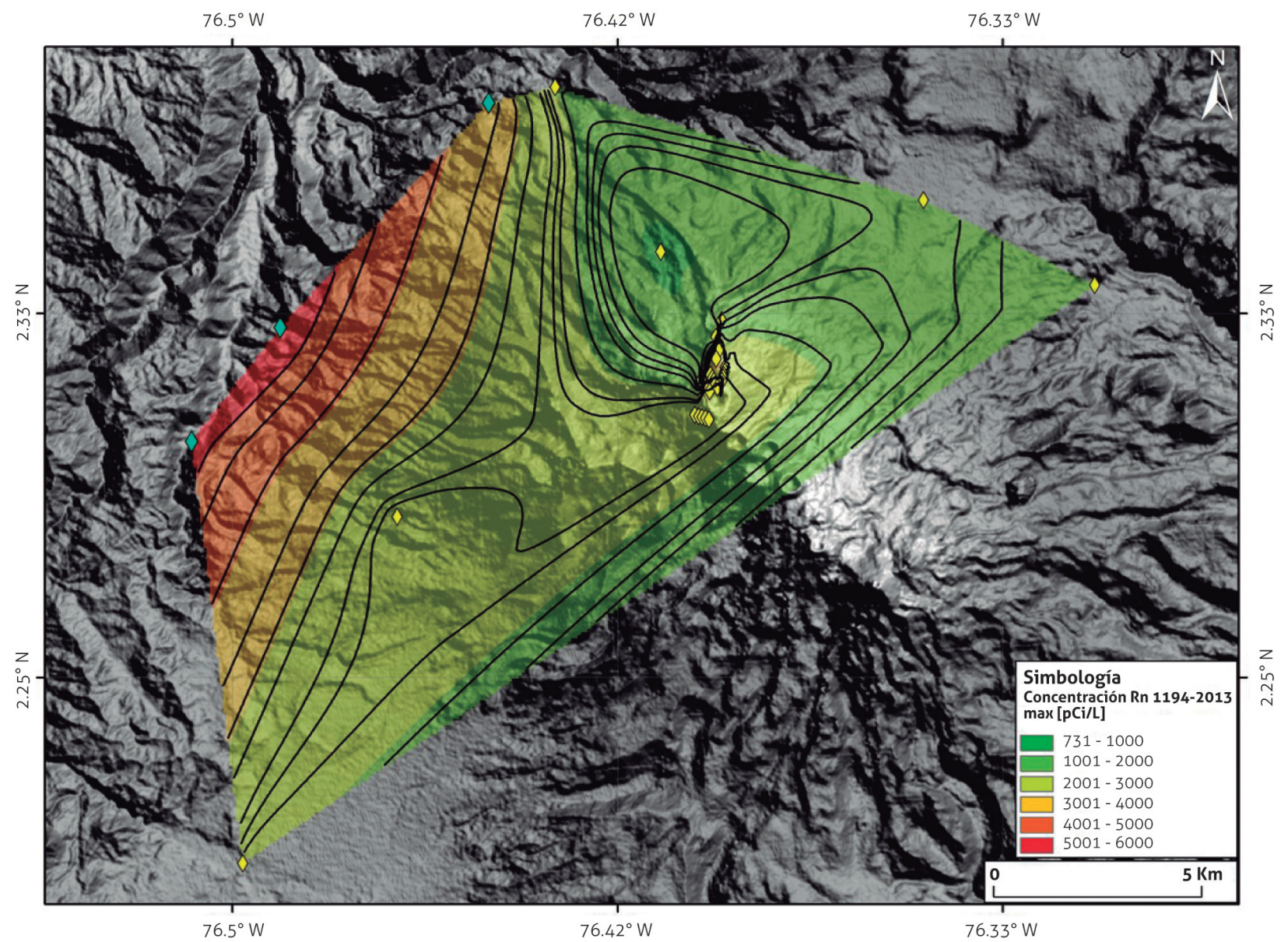

Figura 10. Mapa de anomalías de gas radón en la región del volcán Puracé Fuente: autores

\section{Conclusiones}

En este estudio se pudo establecer que en más de un $80 \%$ de los casos existe un aumento en la concentración de gas radón por encima de $3200 \mathrm{pCi} / \mathrm{L}$ antes de la ocurrencia de sismos con magnitudes mayores a 2,8 ( $M L>)$, en un radio de $35 \mathrm{~km}$ en la zona de los Coconucos. Las anomalías ocurren en un lapso de 3 a 84 días antes del sismo de $\mathrm{ML}>2,8$, con 31 días como promedio. Este hallazgo permite concluir que el monitoreo de gas radón en la zona de estudio es una herramienta útil para pronosticar la ocurrencia de sismos, por lo que se puede convertir en una herramienta adicional para el monitoreo del volcán Puracé y otros volcanes de la región, ya que sismos de magnitud considerable pueden desestabilizar el sistema volcánico, lo que permite usar las mediciones de gas radón como un parámetro de alerta temprana.
La selección de estaciones de medición de gas radón en suelo para el análisis es un factor importante a la hora de detectar posibles relaciones con actividad sísmica tectónica local o volcánica. Igualmente, la ubicación de las mismas es determinante para el análisis e interpretación de los datos.

\section{Agradecimientos}

Los autores desean agradecer al grupo de trabajo del OVSPop por su apoyo y discusiones. Este trabajo se realizó en el marco del proyecto "Investigación y monitoreo de la actividad volcánica de Colombia”, proyecto AME1306 de la Dirección de Amenazas Geológicas del Servicio Geológico Colombiano (SGC). 


\section{REFERENCIAS BIBLIOGRÁFICAS}

Aubert, M. y Baubron, J. (1988). Identification of a hidden thermal fissure in a volcanic terrain using a combination of hydrothermal convection indicators and soil atmosphere analysis. Journal of Volcanology and Geothermal Research, 35(3), 217-225. Doi: 10.1016/03770273(88)90018-2.

Baubron J., Allard P. y Toutain, J. (1990). Diffuse volcanic emissions of carbon dioxide from Vulcano island, Italy. Nature, 344(6261), 51-53. Doi: 10.1038/344051a0.

Baubron, J., Allard, P., Sabroux, J., Tedesco, D. y Toutain, J. (1991). Soil gas emanations as precursory indicators of volcanic eruptions. Journal of the Geological Society, 148(3), 571-576. Doi: 10.1144/gsjgs.148.3.0571.

Berelson, W., Hammond, D. y Fuller, C. (1982). Radon-222 as a tracer for mixing in the water column and benthic exchange in the southern California borderland. Earth and Planetary Science Letters, 61(1), 41-54. Doi: 10.1016/0012-821X (82)90036-X.

Cable, J., Burnett, W., Chanton, J. y Weatherly, G. (1996). Estimating groundwater discharge into the northeaster Gulf of Mexico using radon-222. Earth and Planetary Science Letters, 144(3-4), 591-604. Doi: 10.1016/ S0012-821X (96)00173-2.

Chiodini, G., Frondini, F. y Raco, B. (1996). Diffuse emission of $\mathrm{CO}_{2}$ from the Fossa crater, Vulcano Island (Italy). Bulletin of Volcanology, 58(1), 41-50. Doi: 10.1007/s004450050124.

Cioni, R., Corazza, E. y Marini L. (1984). The gas/steam ratio as indicator of heat transfer at the Solfatara fumaroles, Phlegraean Fields (Italy). Bulletin Volcanologique, 47(2), 295-302. Doi: 10.1007/BF01961560.

Connor, C., Hill, B., La Femina, P., Navarro, M. y Conway, M. (1996). Soil ${ }^{222} \mathrm{Rn}$ pulse during the initial phase of the June-August 1995 eruption of Cerro Negro, Nicaragua. Journal of Volcanology and Geothermal Research, 73(1-2), 119-127. Doi: 10.1016/0377-0273(96) 00020-0.

Cox, M. (1980). Ground radon survey of geothermal areas in Hawaii. Geophysical Research Letters, 7(4), 283-286. Doi: 10.1029/GL007i004p00283.

Cox, M. y Cuff, K. (1981). Monitoring of ground radon concentrations at Kilauea Volcano, Hawaii. Abstract. Tokyo: IAVCEI Symp. Arc Volcanism.
Cox, M., Cuff, K. y Thomas, D. (1980). Variations of ground radón concentrations with activity of Kilauea volcano, Hawaii. Nature, 288(5786), 74-76. Doi: 10.1038/288074a0.

Crenshaw, W., Williams, S. y Stoiber, R. (1982). Fault location by radon and mercury detection at an active volcano in Nicaragua. Nature, 300(5890), 345-346. Doi: $10.1038 / 300345 \mathrm{a} 0$.

Cruz Reina, S. de la, Mena, M., Segovia, N., Chalot, J., Seidel, J. y Monnin, M. (1985). Radon emanometry in soil gases and activity in ashes from El Chichón volcano. Pure and Applied Geophysics, 123(3), 407-421. Doi: 10.1007/BF00880740.

D’alessandro, W. y Parello, F. (1997). Soil gas prospection of $\mathrm{He},{ }^{222} \mathrm{Rn}$ and $\mathrm{CO}_{2}$ : Vulcano Porto area, Aeolian Islands, Italy. Applied Geochemistry, 12(2), 213-224. Doi: 10.1016/S0883-2927(96)00066-2.

Del Pezzo, E., Gasparini, P., Mantovani, M, Martini, M., Capaldi, G. et al. (1981). A case of correlation between Rn anomalies and seismic activity on a volcano (Vulcano Island, Southern Tyrrhenian Sea). Geophysical Research Letters, 8(9), 962-965. Doi: 10.1029/ GL008i009p00962.

Dobrovolsky, I., Zubkov, S. y Miachkin, V. (1975). Estimation of the size of earthquake preparation zones. Pure and Applied Geophysics, 117(5), 1025-1044. Doi: 10.1007/BF00876083.

Fleischer, R. (1988). Radon in the environment-Opportunities and hazards. International Journal of Radiation Applications and Instrumentation. Part D. Nuclear Tracks and Radiation Measurements, 14(4), 421-435. Doi: 10.1016/1359-0189(88)90001-5.

Fleischer, R., Hart, H. y Mogro Campero, A. (1980). Radon emanation over an ore body: search for long-distance transport of radon. Nuclear Instruments and Method, 173(1), 169-181. Doi: 10.1016/0029-554X (80)90584-4.

Fleischer, R. y Mogro Campero, A. (1985). Association of subsurface radon changes in Alaska and northeaster United States with earthquakes. Geochimica et Cosmochimica Acta, 49(4), 1061-1071. Doi: 10.1016/00167037(85)90319-9.

Fuentes, C., Mazadiego, L., Llamas, J., Elorza, F., Chacón, E. y Vela, A. (1995). Aplicación de la emanometría al campo de la modelización geológica granítica para la 
detección de fracturas permeables. Ponencia. VI Congreso de Geoquímica de España.

Garzón, G. (1998). El radón: manual teórico-práctico. Informe. Bogotá: Ingeominas.

Garzón, M. (1987). Posibilidades que ofrece la medida del radón para la prospección de yacimientos de hidrocarburos (tesis doctoral). Oviedo: Universidad de Oviedo.

Gasparini, P. y Mantovani, M. (1978). Radon anomalies and volcanic eruptions. Journal of Volcanology and Geothermal Research, 3(3-4), 325-341. Doi: 10.1016/0377-0273(78)90042-2.

Gil, F. (1998). Procesamiento de sismos volcano-tectónicos asociados con la falla Moras en el área del volcán Puracé. Informe interno. Manizales y Popayán: Ingeominas.

Grosse, E. (1926). El Terciario Carbonífero de Antioquia. Berlín: Dietrich Reimer.

Guedalia, D., Ntsila, A., Druilhet, A. y Fontan, J. (1980). Monitoring of the atmospheric stability above an urban and suburban site using sodar and radon measurements. Journal of Applied $\mathrm{Me-}$ teorology and Climatology, 19(7), 839-848. Doi: 10.1175/1520-0450(1980)019<0839:MOTASA $>2.0$. $\mathrm{CO} ; 2$.

Hauksson, E., J.G. and Goddard (1981): Radon earthquake precursor studies in Iceland. Journal of Geophysical Research: Solid Earth, 86(B8), 7037-7054. Doi: 10.1029/JB086iB08p07037.

Heiligmann, M., Stix, J., WilliamsJones, G., Sherwood, B. y Garzón, G. (1997). Distal degassing of radon and carbon dioxide on Galeras volcano, Colombia. Journal of Volcanology and Geothermal Research, 77(1-4), 267-283. Doi: 10.1016/S0377-0273(96)00099-6.

Hunyadi, I., Hakl, J., Lenart, L., Geczy, G. y Csige, I. (1991). Regular subsurfase radón measurements in Hungarian karstic regions. International Journal of Radiation Applications and Instrumentation. Part D. Nuclear Tracks and Radiation Measurements, 19(1-4), 321-326. Doi: 10.1016/1359-0189(91)90204-U.

Junge, C. (1963). Air chemistry and radiochemistry. New York y London: Academic Press.

King, C. (1980). Episodic radon changes in subsurface soil gas along active faults and possible relation to ear- thquakes. Journal of Geophysical Research: Solid Earth, 85(B6), 3065-3078. Doi: 10.1029/JB085iB06p03065.

King, C. (1985). Impulsive radon emanation on a creeping segment of San Andreas Fault, California. Pure and Applied Geophysics, 122(1-4), 340-352. Doi: 10.1007/BF00874603.

Kotrappa, P., Dempsey, J., Hickey, J. y Stieff, L. (1988). An electret passive environmental 222-Rn monitor based on ionization measurement. Health Physics, 54(1), 47-56.

Kotrappa, P., Dempsey, J., Ramsey, R. y Stieff, L. (1990). A practical E-PERM (electret passive environmental radon monitor) system for indoor ${ }^{222} \mathrm{Rn}$ measurement. Health Physics, 58(4), 461-467.

Londoño, J. (2013). Un método para la detección de anomalías de gas radón en suelo asociadas con la ocurrencia de sismos. Informe interno. Bogotá: Servicio Geológico Colombiano.

Londoño, J. (2009). Radon and $\mathrm{CO}_{2}$ emissions in different geological environments as a tool for monitoring volcanic and seismic activity in central part of Colombia. Boletín de Geología, 31(2), 83-95.

Marquínez, G., Rodríguez, Y., Terraza, R. y Martínez, M. (2003). Geología de la plancha 365-Coconuco. Memorias. Bogotá: Ingeominas.

Martinelli, G. y Ferrari, G. (1991). Earthquake forerunners in a selected area of Northern Italy: recent developments in automatic geochemical monitoring. Tectonophysics, 193(4), 397-410. Doi: 10.1016/00401951(91)90348-V.

Mazadiego, L. (1994). Desarrollo de una metodología para la prospección geoquímica en superficie de combustibles fósiles (tesis doctoral). Universidad Politécnica de Madrid, Madrid, España.

Meza Maldonado, L., Inguaggiato, S., Jaramillo, M., Garzón Valencia, G. y Mazot, A. (2017). Volatiles and energy released by Puracé volcano. Bulletin of Volcanology, 79(12), 84. Doi: 10.1007/s00445-017-1168-y.

Monsalve, M. y Pulgarín, B. (1993). Mapa preliminar de amenaza volcánica potencial del volcán Puracé. Revista Ingeominas, 2, 3-27.

Monsalve, M. y Pulgarín, B. (1999). Cadena volcánica de los Coconucos: centros eruptivos y productos recientes. Boletín Geológico, 37(1-3), 17-51. 
Nishizawa, S., Igarashi, G., Sano, Y., Shoto, E., Tasaka, S. y Sasaki, Y. (1998). Radon, Cl- and SO42- anomalies in hot spring water associated with the 1995 earthquake swarm off the east coast of the Izu Peninsula, central Japan. Applied Geochemistry, 13(1), 89-94. Doi: 10.1016/S0883-2927(97)00058-9.

Parello, F., D’alessandro, W., Bonfanti, P. y Dongarra, G. (1995). Subsurface gases in selected cities of the Mount Etna área (Sicily). Acta Vulcanológica, 7(1), 35-42.

Pulgarín, B. y Agudelo, A. (2003). Entorno geológico de los sismos sentidos en las poblaciones de Sotará, Coconucos, La Sierra, Rosas, Timbío, Popayán, Inzá y Benalcázar los días 7 de enero y 2 de febrero de 2003. Informe interno. Popayán: Ingeominas.

Pulgarín, B., Monsalve, M., Arcila, M. y Cepeda, H. (1994). Actividad histórica y actual del volcán Puracé, Colombia. Boletín Geológico, 34(2-3), 39-53.

Purtscheller, F., Pirchl, T., Sieder, G., Stingl, V., Tessadri, T. y Brunner, P. (1995). Radon emanation from giant landslides of Koefels (Tyrol, Austria) and Langtang Himal (Nepal). Environmental Geology, 26(1), 32-38. Doi: 10.1007/BF00776029.

Purtscheller, F., Stingl, V., Brunner, P. y Ennemoser, O. (1994). The Tsergo Ri landslide (Langtang Himal): A case study of radon emanation from giant landslides. Journal of Nepal Geological Society, 10, 102-104.

Orrego, A. (1977). Geología y geoquímica del área mineralizada de El Pisno, Cauca. Publicación Geológica Especial, vol. 10. Ingeominas.

Orrego, A. y París, G. (1991). Cuadrángulo N-6, Popayán: geología, geoquímica y ocurrencias minerales. Escala 1:100.000. Popayán: Ingeominas.

Ramola, R., Singh, S. y Virk, H. (1988). Uranium and radon estimation in some water samples from $\mathrm{Hi}$ malayas. International Journal of Radiation Applications and Instrumentation. Part D. Nuclear Tracks and Radiation Measurements, 15(1-4), 791-793. Doi: 10.1016/1359-0189(88)90252-X.

Sato, T., Wakita, H., Notsu, K. y Igarashi G. (1992). Anomalous hot spring water changes: Possible precursors of the 1989 volcanic eruption off the east coast of the Izu Peninsula. Geochemical Journal, 26(2), 73-83.
Segovia, N., Cruz Reyna, S. de la, Mena, M., Seidel, J. y Monnin, M. (1991). Radon-222 as an indicator of geothermal reservoirs behaviour. International Journal of Radiation Applications and Instrumentation. Part D. Nuclear Tracks and Radiation Measurements, 19(1-4), 401-404. Doi: 10.1016/1359-0189(91)90225-7.

Seidel, J. y Monnin, M. (1984). Mesures de radon-222 dans le sol de l'Etna (Sicile): 1980-1983. Bulletin Volcanologique, 47(2), 1071-1077. Doi: 10.1007/ BF01952363.

Seidel, H., Freeman, S., Seto, H. y Knowles J. (1988). Phosphonate biosynthesis: Isolation of the enzyme responsible for the formation of a carbon-phosphorus bond. Nature, 335(6189), 457-458. Doi: 10.1038/335457a0.

Shapiro, M., Melvin, J., Tombrello, T. y Whitcomb, J. (1980). Automated radon monitoring at a hard rock site in the southern California traverse ranges. Journal of Geophysical Research, 85(B6), 3058-3064. Doi: 10.1029/JB085iB06p03058.

Teng, T. (1980). Some recent studies on groundwater radon content as an earthquake precursor. Journal of Geophysical Research, 85(B6), 3089-3099. Doi: 10.1029/JB085iB06p03089.

Thomas, D., Cuff, K. y Cox, M. (1986). The association between ground gas radon variations and geologic activity in Hawaii. Journal of Geophysical Research, 91(B12), 12186-12198. Doi: 10.1029/ JB091iB12p12186.

Toutain, J., Baubron, J., Le Bronec, J., Allard, P., Briole, P., Marty, B. et al. (1992). Continuous monitoring of distal gas emanations at Vulcano, southern Italy. Bulletin of Volcanology, 54(2), 147-155. Doi: 10.1007/ BF00278004.

Ulomov, V. y Mavashev, B. (1971). The Tashkent Earthquake of 26 April, 1966. Tashkent: Akademii nauk Usbekskoi SSR.

Virk, H. y Singh, B. (1993). Radon anomalies in soilgas and groundwater as earthquake precursor phenomena. Tectonophysics, 227(1-4), 215-224. Doi: 10.1016/0040-1951(93)90096-3.

Wakita, H., Nakamura, Y. y Sano, Y. (1988). Short-term and intermediate-term geochemical precursors. Pure and Applied Geophysics, 126(2-4), 267-278. Doi: 10.1007/BF00878999. 
Whitehead, N., Gingrich, J. y Fisher, J. (1983). A New Zealand test of the track-etch method of prospecting for geothermal steam. Journal of Volcanology and Geothermal Research, 15(4), 339-354. Doi: 10.1016/03770273(83)90106-3.
Wilkening, M. y Watkins, D. (1976). Air exchange and ${ }^{222} \mathrm{Rn}$ concentrations in the Carlsbad Caverns. Health Physics, 31(2), 139-145. 